Trinity University

Digital Commons @ Trinity

Psychology Faculty Research

Psychology Department

2009

\title{
Law Enforcement Preferences for PTSD Treatment and Crisis Management Alternatives
}

Carolyn Becker

Trinity University, cbecker@trinity.edu

Glenn Meyer

Trinity University, gmeyer@trinity.edu

J. S. Price

M. M. Graham

Ashley Arsena

Trinity University

See next page for additional authors

Follow this and additional works at: https://digitalcommons.trinity.edu/psych_faculty

Part of the Psychology Commons

Publication Details

Behaviour Research and Therapy

\section{Repository Citation}

Becker, C.B., Meyer, G., Price, J.S., Graham, M.M., Arsena, A., Armstrong, D.A., Ramon, E. (2009). Law enforcement preferences for PTSD treatment and crisis management alternatives. Behaviour Research and Therapy, 47(3), 245-253. doi: 10.1016/j.brat.2009.01.001

This Post-Print is brought to you for free and open access by the Psychology Department at Digital Commons @ Trinity. It has been accepted for inclusion in Psychology Faculty Research by an authorized administrator of Digital Commons@ Trinity. For more information, please contact jcostanz@trinity.edu. 


\section{Authors}

Carolyn Becker, Glenn Meyer, J. S. Price, M. M. Graham, Ashley Arsena, D. A. Armstrong, and Elizabeth Ramon 
Running Head: POLICE PTSD TREATMENT

Law Enforcement Preferences for PTSD Treatment and Crisis Management Alternatives

Carolyn Black Becker, Glenn Meyer

Trinity University, San Antonio, TX

John S. Price, Melissa M. Graham

San Antonio Police Department, San Antonio, TX

Ashley Arsena

Trinity University, San Antonio, TX

David A. Armstrong

McNeese State University, Lake Charles, LA

Elizabeth Ramon

Trinity University, San Antonio, TX

Address correspondence to Carolyn Becker, Ph.D., Department of Psychology, Trinity University, One Trinity Place, San Antonio, TX 78212-7200. Phone 210-999-8326; Fax 210-9998386; Email: carolyn.becker@trinity.edu

In press: Behaviour Research and Therapy 


\begin{abstract}
Evidence-based treatments (EBT) for posttraumatic stress disorder (PTSD) remain underutilized. Analog research, however, indicates that patients may be more amenable to receiving EBT for PTSD than utilization rates suggest. This study sought to extend previous studies by investigating PTSD treatment preferences among law enforcement individuals (i.e., active duty officers, cadets, criminal justice students). We asked 379 participants, with varying trauma histories, to read a police traumatic event and imagine they had developed PTSD. Participants rated the credibility of six treatment options which they might encounter in a treatment setting, and chose their most and least preferred treatments. Next, they evaluated a widely used debriefing intervention aimed at preventing PTSD. Almost $90 \%$ of participants chose exposure or cognitive processing therapy as their first or second most preferred treatment, and they rated these interventions as significantly more credible than the other 4 treatment options. The sample showed ambivalence regarding the perceived efficacy of debriefing but found the rationale credible. This study supports previous analog research indicating that patients may be more interested EBT than indicated by utilization rates, and suggests that law enforcement departments should consider offering EBT to officers who develop PTSD.
\end{abstract}

Keywords: Evidence-based, CBT, PTSD, exposure, CISD, police 


\subsection{Introduction}

Dissemination of evidence-based treatment (EBT) remains a significant challenge for the field of clinical psychology (Young, Connolly, \& Lohr, 2008). For instance, research indicates that one of the most efficacious treatments for posttraumatic stress disorder (PTSD), exposure therapy (Foa, Keane, \& Friedman, 2000), continues to be underutilized in clinical practice (Becker, Zayfert, \& Anderson, 2004; Foy et al., 1996; Rosen et al., 2004). This trend is disturbing given that research suggests that cognitive behavioral treatments (CBT), such as exposure, can substantially reduce PTSD symptoms and also can be successfully delivered by community therapists (see Cahill, Foa, Hembree, Marshall, \& Nacash, 2006 for discussion). It should be noted that dissemination findings are not unique to CBT for PTSD. For example, research also indicates that therapists do not use exposure for other anxiety disorders (Freiheit, Vye, Swan, \& Cady, 2004).

Clinical underutilization of EBT may result from a variety of therapist and patient factors (Becker, Darius, \& Schaumberg, 2007). Therapist factors that may contribute to EBT underuse, for instance, include lack of training or comfort in delivering EBT, perceptions about interventions, and/or personal preferences for certain types of therapy. Patient factors include, but are not limited to, credibility of treatment rationale, anticipation of discomfort, and/or willingness to tolerate discomfort. Therapist factors and patient factors also may interact. For example, a therapist may lack comfort in delivering a given EBT - possibly because of lack of training. If a patient finds the description of the treatment anxiety provoking, the therapist and patient may collude in avoiding a challenging yet potentially efficacious treatment strategy.

Although previous research provides support for the role of therapist factors in limiting use of EBT for PTSD (Becker et al., 2004; Najavits, 2006; Rosen et al., 2004), prior studies have not clearly implicated patient factors in limiting use of EBT. For instance, three analog studies conducted with student samples (Becker et al., 2007; Tarrier, Liversidge, \& Gregg, 2006; Zoellner, Feeny, Cochran, \& Pruitt, 2003) consistently found that a sizeable majority of 
participants ranked CBT interventions with a strong evidence base (including those that involved exposure) as highly preferred over other forms of psychotherapy and/or medication. This finding was replicated in one non-collegiate sample of women who responded to advertisements seeking females with trauma histories (Angelo, Miller, Zoellner, \& Feeny, 2008). Seventy-one percent of the Angelo et al. sample had experienced a Criterion A event and 53\% met PTSD criteria. Approximately $82 \%$ of participants chose exposure when given a forced choice option of exposure, versus $13 \%$ for sertraline and $6 \%$ for no treatment. In this study, treatment mechanism (e.g., I need to talk about what happened) was the most commonly cited reason for choosing a treatment, followed by treatment efficacy and health concerns.

To our knowledge, aside from Angelo et al. (2008), no study has examined a predominately non-collegiate sample's perceptions of PTSD treatment options. Furthermore, because Angelo et al. only gave participants the option of one type of psychotherapy (exposure) versus medication or no treatment, no study has explored perceptions about different types of psychotherapy along with medication in a non-collegiate population at high risk for traumatic events. Thus, Angelo et al.'s results may indicate that participants preferred psychotherapy to medication and that the rationale for psychotherapy made more sense than that for medication. In sum, this study does not provide information about perceptions of different types of psychotherapy, with varying levels of empirical support, in a largely non-collegiate sample. Law enforcement professionals may be at risk for significant exposure to traumatic events. Such events include, but are not limited to, line of duty deaths, serious injury to police, school or workplace shootings, witnessing suicides, police shooting individuals in the line of duty, familial violence, handling dead bodies, and mass fatality terrorist attacks such as $9 / 11$ (Miller, 2006; Volanti et al., 2006). Although law enforcement culture may lead to a perception that officers can psychologically cope with these intense, repeated stressors (Wright, Borrill, Teers, \& Cassidy, 2006), research regarding post-trauma symptomatology suggests otherwise. For example, Dowling, Moynihan, and Genet (2006) reported 68\% of 9/11 responding officers 
reported at least one disaster related stress symptom 15-27 months later, with at least $20 \%$ having significant difficulties such that they were advised to seek further assistance. Wright et al. found that $37 \%$ of prison officers met criteria for PTSD 3-7 months after a death in custody episode, and Violanti et al. (2006) found that almost 30\% of a random stratified sample of officers from an urban police department endorsed moderate to severe PTSD symptoms. Law enforcement personnel who experience stressful or traumatic incidents also may be more likely to leave the job within several years, and appear to struggle with suicide, substance abuse, spousal abuse and divorce at rates that are substantially higher than seen in the general population (Dowling, et al. 2006; Miller, 2006). Thus, it is not surprising that a professional and popularized literature now exists with the aim of addressing the needs of police coping with stressful events (e.g. Artwohl \& Christensen, 1997; Klinger, 2004; Kates, 1999).

As noted above, however, law enforcement personnel may view themselves as immune to stressful events and are sometimes reluctant to seek mental health services. As Miller (2006, p.96) notes: whereas some officers are amenable to psychotherapy, "other cops would rather swim through boiling oil than sit in a psychologists' office." According to Miller, seeing a therapist may not fit into the paramilitary culture of the police because it indicates weakness and also may not be attractive to the socio-economic class of people who often become officers. Finally, some officers worry about confidentiality and possible threats to their job security (Dowling, 2006; Miller, 2006). In sum, a variety of factors may limit officer help seeking after a traumatic event. For this reason, it could be of practical importance to determine what interventions, processes or therapies would be the most and least attractive to law enforcement personnel. For instance, officers might show similar preferences to previously studied samples (i.e., Angelo et al., 2008; Becker et al., 2007; Tarrier et al., 2006; Zoellner et al., 2003). If officers do show a preference for EBT, this preference (along with the evidence-base) could be used to encourage police department therapists to offer EBT and to design departmental programs and 
guidelines for this population. On the other hand, if officers show an aversion to EBT, then a barrier to bringing EBT to police departments becomes better clarified.

The purpose of this study was to extend Becker et al. (2007) by investigating PTSD treatment preferences among active law enforcement personnel, cadets, and students planning to enter the law enforcement field. We included a broad array of law enforcement individuals so as to obtain a moderately large sample that represents some of the breadth in this field. The three subgroups also offer the opportunity to explore on a very preliminary basis if those with real experience with police work have different views than those without such experience.

Becker et al. (2007) examined seven therapeutic options: exposure, a general form of CBT, sertraline (i.e., Zoloft), Eye Movement Desensitization and Reprocessing therapy (EMDR); psychodynamic therapy, and two pseudoscientific therapies (i.e., Thought Field Therapy and My Therapy Buddy). The present study relied on a similar core list: exposure; sertraline, EMDR and psychodynamic therapy. Although EMDR was not well received in previous analog studies (e.g., Becker et al., Tarrier et al., 2006), we included EMDR because it is an EBT that appears popular among therapists, despite significant controversy about its mechanism of action. In this study, we modified the general CBT description in Becker et al. to instead describe Cognitive Processing Therapy (CPT; Resick \& Schnicke, 1992; Resick, Nishith, Weaver, Astin, \& Feuer, 2002), one of the specific, well-studied efficacious forms of CBT for PTSD. We did this in order to a) examine if there was any preference differences for two of the most well-studied forms of CBT for PTSD (i.e., exposure and CPT), and b) because agencies such as police departments may find it easier to bring in trainers for specific forms of CBT should they choose to adopt such interventions. In addition to changing the CBT intervention, we dropped the two pseudoscientific treatments used in Becker et al. because we wanted to explore officer attitudes about other options that are discussed in the police/trauma literature. Given that participants received no compensation for participation, we felt it prudent to reduce participant burden as much as 
possible. The added options included Brief Eclectic Psychotherapy (BEP) and Critical Incident Stress Debriefing (CISD).

BEP combines components of cognitive-behavioral and psychodynamic therapy to form a brief, eclectic treatment for traumatic symptoms (Gersons, Carlier, Lamberts, \& van der Kolk, 2000). BEP has been recommended as useful for police officers (Lindauer et al., 2005), and one trial found it superior to waitlist in officers seeking treatment for PTSD (Gersons et al., 2000). CISD is an intervention requiring only one or two sessions of debriefing shortly after a traumatic event. The goal of CISD is to prevent the emergence of full-blown psychopathology; it typically is based in group work run with high-risk occupational groups, although individual debriefing sessions may be used (Adler et al., 2008; Devilly, Gist, \& Cotton, 2006). Use of CISD is widespread (Devilly et al.; Gist \& Devilly, 2002) and CISD is popular with the law enforcement community (Miller, 2006). CISD includes reconstructing, venting, and normalizing the traumatic event along with some psychoeducation. Adler et al. (2008, p. 253) suggest that CISD is attractive to organizations (e.g., military and paramilitary) because it is sensitive to work cultures, uses peer processes, and is viewed as providing an opportunity for organization members to share their responses to traumatic events. CISD also may be viewed by some as meeting organizational duty of care requirements (Devilly et al.). Finally, because CISD is an immediate and often mandated group intervention, it may not share the perceived stigma of later occurring psychotherapies. Despite these potentially positive features and the ongoing extensive use of CISD, however, research support for this intervention remains limited and troubling. Much of the supporting research suffers major methodological problems (Devilly et al.), and a recent meta-analysis found that CISD did not improve outcome following a traumatic event (van Emmerik, Kamphuis, Hulsbosch, \& Emmelkamp, 2002). Finally, one of the most recent well controlled studies of CISD among US peacekeepers did not support its use (Adler et al., 2008). Secondary to the problematic research base, researchers, practitioners, consensus panels, and evidence-based guidelines have cautioned that use of CISD be limited or viewed as 
contraindicated (Gist \& Devilly, 2002). Unfortunately, these recommendations have not been followed given that, as noted above, CISD remains widely used (Gist \& Devilly).

Based on the past analog studies discussed above, we hypothesized that individuals in law enforcement would show a preference for exposure and CPT over other interventions such as medication, psychodynamic therapy and BEP. Given low preference ratings for EMDR in two previous studies (Becker et al., 2007; Tarrier et al., 2006) we hypothesized that participants would show little interest in EMDR despite the fact that it has received empirical scrutiny and support (Rothbaum, Astin, \& Marsteller, 2005). Our research design focused on having officers respond to a scenario situation in which they developed PTSD. Because CISD is not a treatment for PTSD but rather an intervention designed to prevent later onset of psychopathology, we could not directly compare this intervention with the others in terms of treatment preferences. We did hypothesize, however, that participants would respond at least somewhat favorably to the general rationale for CISD given its popularity. Because we are not aware of any literature that would lead to predictions about differences between law enforcement students, cadets, and active duty officers, we made no hypotheses about these groups aside from hypothesizing a greater number of Criterion A events for active duty officers, secondary to their time in the field. We also planned to collapse the data if we found no significant evidence of differences in these sub-groups.

\subsection{Method}

\subsubsection{Participants}

The sample for this study $(N=379)$ comprised 99 criminal justice (CJ) students enrolled in a university located in the southeastern section of the United States, 108 cadets recruited from the police department of a large southwestern city, 156 active duty law enforcement officers (e.g., hostage negotiators, park police, detective, patrolpersons etc.) from the same large southwestern city, 12 law enforcement officers from a small city in the midwest, and 4 police officers from a small university campus. The study was approved by the Trinity University 
Institutional Review Board and, as needed, appropriate agencies/individuals (e.g., chief of police). Seventy-one percent of the participants were male, and $29 \%$ were female. Fifty one percent of participants reported Caucasian ethnicity, 38\% Latino/Hispanic, and 10\% African American. Regarding educational status, $65 \%$ of participants had completed some college or held an Associates degree and 23\% had completed their Bachelors degree or beyond. The mean age was $32.00(S D=10.36)$.

\subsubsection{Materials}

1.2.2.1 Treatment Descriptions. Descriptions included background and efficacy information along with procedures, usual duration, and possible side effects for each of six main treatment options plus CISD. We sought to offer participants a choice of treatment interventions that varied both in terms of orientation (e.g., cognitive behavioral, psychodynamic, pharmacologic) and the degree of empirical support, so as to represent the range of treatment options that law enforcement individuals with PTSD might encounter. Treatment options included three treatments with robust empirical support, two of which were psychological (exposure therapy and CPT) and one of which was pharmacological (sertraline). As in Becker et al. (2007), we also included two forms of psychotherapy that appear popular among therapists despite being somewhat controversial regarding mechanism of action and/or having less empirical support than exposure and CPT (i.e., EMDR and psychodynamic psychotherapy).We also included one treatment (e.g., BEP) that appears to be gaining some popularity among clinicians who treat law enforcement officers (Lindauer et al., 2005). As noted above, because of the popularity of CISD, we asked participants to comment on CIDS after ranking their main treatment choices. Because CISD is delivered after a traumatic event, but before someone develops PTSD, it was not appropriate to include it with the treatment options for PTSD.

Treatment descriptions were modified from the ones used in Becker et al. (2007) and were constructed to reflect actual information that might be presented to an individual seeking treatment for PTSD. Based on feedback from colleagues who work with this population, 
modifications were made to make the descriptions easier to read. Aside from these changes, four of the treatment descriptions were identical to those used in Becker et al. (exposure, sertraline, EMDR, and psychodynamic). The exposure and sertraline descriptions also were used by Zoellner et al. (2003). We modified the CBT description used in Becker et al. to make it consistent with a more purely CPT approach. The descriptions for BEP and CISD were created to match the other descriptions in length and style, and were based on the existing literature. The CPT treatment description was shared with a colleague with CPT expertise for feedback. We did not send out the other treatment descriptions for review because four of the descriptions had been reviewed for the Becker et al. study, and because we did not have colleagues who were experts in BEP or CISD. These latter two descriptions were sent to several clinicians, however, for feedback and modified accordingly.

We sought to present all therapies as viable treatment options. All psychological descriptions were approximately of similar length, between 192 (sertraline) to 228 (BEP) words. See Appendix A for detailed treatment descriptions along with word counts.

1.2.2.2 Measures. As in Becker et al. (2007), we assessed participants' opinions of each treatment using a slightly modified version the Credibility Scale (CS; Addis \& Carpenter, 1999), which uses a 1-7 Likert scale ranging from "not at all" to "extremely." Items are summed. This scale assesses the degree to which participants find different treatment descriptions credible. Four of the questions from the CS were unchanged from the original version, with the exception of directing participants to PTSD as opposed to depression. The three modified questions were changed in order to make the questions more straightforward for the participants in this study. We made these changes based on the recommendations of individuals who have extensive experience with this population, and who informed us that participants would appreciate "simple language" that did not appear "too academic." For instance, "How logical does this therapy seem to you?" was changed to "Does this type of therapy make sense to you?" Internal 
consistency for the CS was good with alpha coefficients ranging from a low of .91 for CISD to a high of .96 for psychodynamic therapy in the present sample.

Participants also indicated their first and second choices for treatment as well as their first and second choices for treatments that they would most like to avoid. We did not ask participants to rank all treatment options because we figured they would have stronger opinions about treatments they most and least want, in contrast to those that fall in the middle. By doing this, we sought to avoid a tendency to over-interpret middle ranking positions.

We assessed PTSD using the Posttraumatic Stress Diagnostic Scale (PDS; Foa, Cashman, Jaycox, \& Perry, 1997). The PDS has acceptable diagnostic agreement with interview measures of PTSD (Foa et al., 1997), and it had excellent internal consistency in this sample $(\alpha=.93$ for total symptom severity).

\subsubsection{Procedure}

After a brief verbal introduction to the study, participants were given a study packet containing a written introduction, informed consent forms, an instruction sheet for the study, and a copy of the following trauma scenario:

On a night shift you go to a call of a suspicious male loitering in the streets of a neighborhood. You arrive on the scene and find a male breaking into a car. You instruct him to stop and show you his hands. However, the suspect turns and runs.

You chase him into a dark dead-end ally. The suspect is cornered and turns around, aiming a gun at you. You fire two shots into the suspect's center of mass.

The suspect falls to the ground and drops the gun. You approach the suspect and find that you have fatally shot him. You discover the suspect is a fifteen year-old boy.

At first, you try to forget about the incident but this doesn't work out well.

- After a few months you begin to have nightmares about the incident. 
- Handling your gun becomes very difficult because you feel anxious and have flashbacks to the shooting.

- You don't want to go to work and once there you often find yourself avoiding the area of the shooting.

- Your relationship with your wife and kids has become more difficult.

- Recently, you nearly struck your wife when she woke you up suddenly from a nightmare.

- You find yourself drinking more than usual.

These are common symptoms of Posttraumatic Stress Disorder.

After reading the scenario, participants read each treatment description and immediately completed the associated CS which was printed on the same page as the description.

Descriptions were randomly ordered to prevent order effects. After evaluating all six therapies, participants ranked their top and bottom choices for treatment. Next, participants read the CISD description and completed the CS. They then responded to two final CISD questions. First, they indicated to what degree they thought CISD could prevent need for later treatment using the same Likert scale as used in the CS. Second, they indicated whether or not they believe that law enforcement officers should be required to have CISD after a traumatic event. After this, they were asked about the degree to which they saw themselves developing trauma related stress symptoms at some point in the future and if they developed PTSD, how likely it was that they would seek treatment. We included these to get a general sense of the degree to which the sample saw themselves at risk for PTSD and their general willingness to consider treatment options. These questions were rated on a seven point Likert scale (1-7) ranging from "not very likely" to "very likely". Lastly, participants completed the PDS and demographic questions. Because the study packet was designed to be handed out as a stand alone survey, the final page of the packet contained a take away sheet with mental health resources.

\subsection{Results}




\subsubsection{PTSD Diagnoses and Trauma Histories of Participants}

Thirty-six participants (9.5\%) met DSM-IV criteria for PTSD as assessed by the PDS (Foa et al., 1997). An additional 182 (48.0\%) participants reported experiencing a Criterion A event during their lifetime, but did not meet criteria for PTSD at the time of the study. Primary Criterion A events included serious accidents (19.0\% of total sample), physical assault (12.7\%), natural disasters (12.1\%), sexual assault (9.8\%), combat $(6.1 \%)$, life threatening illness (5.0\%), and other traumas (16.5\%).

Because it could be argued that active law enforcement officers might have experienced a higher rate of traumatic events, we examined the frequency of PTSD diagnosis and Criterion A events among officers, cadets and students separately. For PTSD diagnosis, rates among CJ students $(9.1 \%)$, cadets $(10.2 \%)$, and law enforcement officers $(9.3 \%)$ were virtually identical. An additional $61 \%$ of CJ students reported a lifetime Criterion A event but did not meet PTSD criteria. In contrast to our hypothesis, this compared to $36 \%$ of cadets and $48 \%$ of active duty officers, a statistically significant difference $\chi^{2}(N=343)=13.13, p<.001$. Because rates of PTSD were the same among all groups and because a preliminary examination of treatment preferences showed similar outcomes for the sub-groups, we collapsed the sub-groups for all subsequent analyses.

\subsubsection{Most and Least Preferred Two Treatment Choices}

Treatments were not selected equally as the number one preferred choice by

participants $\chi^{2}(N=375)=264.43, p<.0001$. Consistent with our hypotheses, CPT and exposure were the most preferred therapies, with $36.9 \%$ and $25.9 \%$ of participants selecting these treatments, respectively. The remaining therapies were chosen in the following order: psychodynamic therapy, sertraline, BEP and EMDR (see Table 1). The rank order of the therapies remained unchanged for the second choice with the exception that CPT and exposure 
changed places (i.e., exposure was the most selected second choice). Combined, 329 (87\%) of the participants selected either CPT or exposure as a first or second choice treatment.

To examine the role of trauma history and probable PTSD diagnosis on treatment choice, we examined choice among participants who reported having a Criterion A event and those who met PTSD criteria. Of the 218 participants who reported a Criterion A event (note: this group includes participants with likely PTSD), 39\% chose CPT and $21 \%$ chose exposure as their top therapy choice. As with the total sample, $87 \%$ or 190 of the participants chose either CPT or exposure as a first or second choice. Overall, the ordering of choices remained basically the same as with the total sample.

Thirty-six participants met PTSD criteria. Among this much smaller sample, CPT remained the top choice (39\%), though psychodynamic treatment was ranked as the second top choice (25\%). Only $11 \%$ of individuals with PTSD chose exposure as their top choice. Yet, $53 \%$ rated it as either their first or second choice, a percentage quite comparable to the overall sample (see Table 1). Furthermore, $92 \%$ of individuals who met likely PTSD criteria rated either exposure or CPT as their first or second choice treatment.

Regarding the participants least preferred therapy choice, we once again found that treatments were not equally selected, $\chi^{2}(\mathrm{~N}=374)=340.52, p<.0001$. Medication was the most frequently selected least preferred therapy choice (40\%) followed by EMDR (28\%), BEP (12\%), psychodynamic (9\%), and exposure and CPT (both 4\%). When least and second least choices were combined, $60 \%$ and $57 \%$ reported wanting to avoid EMDR and medication. Less than $10 \%$ of participants selected exposure or CPT as a treatment they wanted least or second least.

\subsubsection{Treatment Rationales}

Mean ratings and standard deviations for the CS for each treatment and CISD are displayed in Table 2. In terms of the main treatment interventions, the three most highly rated were exposure, CPT, and BEP. CISD, however, also was rated highly and was second only to 
exposure in terms of CS scores. EMDR and sertraline were the lowest rated interventions. To investigate the degree to which study participants rated the rationales as equal in terms of credibility, we conducted one within subject repeated measures analysis of variance (ANOVA) using CS scores as the dependent variables. We included CISD in this analysis. Because our hypotheses centered largely on exposure and CPT and because paired comparisons between each intervention would have resulted in 21 follow-up tests, we limited post-hoc comparisons to two sets of simple contrasts, the first between exposure and the remaining treatments and the second between CPT and the remaining treatments. To adjust for multiple comparisons (11 in total) we used an adjusted significance level of .005. For the overall repeated measures ANOVA there was a significant within subjects effect, $F(6,2172)=137.17, p=.0001$, partial $\eta^{2}$ $=.28$, indicating that participants rated the interventions differently in terms of the perceived credibility of the rationales. Simple contrasts indicated that participants rated exposure more highly than each of the other treatments, including CPT, with the exception of CISD. A second round of contrasts that compared CPT to all interventions except exposure indicated that CPT was rated as significantly more credible than all of the interventions except CISD. There was no significant difference between CPT and CISD, which were rated almost identically.

Analyses of CS scores in participants who reported a Criterion A event showed an identical pattern except that the difference between CPT and exposure was no longer significant. Among the much smaller sample of participants who met self-report criteria for PTSD, exposure only remained significantly better rated than sertraline and EMDR. The same pattern was observed for CPT.

\subsubsection{Additional CISD Questions and Likelihood of Developing PTSD}

At the end of the CISD CS, we asked participants to what degree they believed that CISD could prevent the need for later treatment. The mean rating was $3.80(S D=1.74)$ and the median was 4 . Closer examination of this question revealed that a minority of participants (18\%) 
thought this outcome was very likely (i.e., rated a 6 or 7 ) and a minority thought it was very unlikely (27\%). Next we asked whether or not participants thought that law enforcement officers should be required to have CISD after a traumatic event. Even though the description of CISD noted that recent research indicates that CISD may not have any long term effectiveness and even though most participants did not strongly report thinking that CISD would prevent the need for future treatment, $77 \%$ of participants said they did think it should be required.

We also asked participants how likely they thought it was that they would develop PTSD symptoms (rated on a 1-7 Likert scale from "not very likely" to "very likely"). The mean score was $3.95(S D=1.74)$. The median and mode were both 4.00 . The mean rating for the question asking whether they would seek treatment if they did develop PTSD was $5.05(S D=1.57)$. The median was 5.00 and the mode was 6.00 . Only a minority of participants responded to this question, however $(n=111)$. This was the only question with significant missing data.

\subsection{Discussion}

Although dissemination of EBT for PTSD has been less than satisfactory to date, previous research regarding analog patient preferences for PTSD treatment indicates that patient factors may play a smaller role than expected given that studies have consistently shown a patient preference for EBT for PTSD. Past research was somewhat limited, however, in that most studies were conducted with university samples. The purpose of this study was to examine a predominately non-collegiate sample at high risk for traumatic events. Consistent with our hypotheses, treatments were not selected equally. Law enforcement individuals, comprised of active duty officers, cadets and CJ students, showed a strong preference for EBT for PTSD with $87 \%$ of the sample choosing either CPT or exposure as either a first or second choice. This is an important finding because it suggests that if police departments offered both exposure and CPT then officers would not only have access to EBT, they also would have access to treatment modalities that appear preferred. Importantly, this finding held even when 
we examined participants who had experienced a Criterion A event as well as those who appeared to meet criteria for PTSD.

Results from this study also indicated that exposure and CPT were the two treatments that were rated as most credible. Comparisons between credibility scores showed that both exposure and CPT were rated as significantly more credible than all other treatments for PTSD (note: this does not include CISD which is not a treatment). The existing police and trauma literature suggests that officers may be reluctant to seek treatment for PTSD. We could find no evidence in the literature, however, that officers are routinely offered EBT such as CPT and exposure. Thus, it is conceivable that providing officers with treatment that both works and is viewed as credible and preferred may increase officers' willingness to seek treatment. Interestingly, after reading through all of the rationales, officers who responded to our question about likelihood of treatment seeking, showed an overall willingness to seek treatment with a mean and median score of 5.00 on a 1-7 Likert scale. Indeed, $46 \%$ of those who responded indicated that they were likely or very likely to seek treatment (rating 6 or 7 ) should they develop PTSD. We also should note, however, that less than $50 \%$ of participants responded to this question, and we had no significant missing data problems with any other question. Although it is impossible to know how to interpret missing data, one possible explanation for the low response rate on this question is that many participants were still reluctant to seek treatment but did not want to indicate this on their questionnaire for some reason.

In terms of differences in preferences for CPT versus exposure, we believe that the literature shows no clear pattern. In the present study, for instance, CPT was chosen as the top choice somewhat more than exposure but exposure was chosen just barely more often than CPT for the top two choices. Exposure also was rated as significantly more credible, although the actual magnitude of difference was fairly small. In our opinion, there is little to be gained at this point in trying to compare these two different forms of CBT against one another in terms of palatability. Rather we would argue that it may make sense for police departments to offer both 
exposure and CPT to officers needing treatment for PTSD given that our results indicate that this could match the preferences of approximately $90 \%$. Although there is little support for the benefit of treatment matching in outcome, allowing officers some choice in which form of EBT they receive could increase their sense of control over treatment and make treatment seeking more acceptable. This supposition, however, would need to be tested.

After CPT and exposure, participants showed the greatest interest in psychodynamic therapy followed by sertraline. These results are identical to those from our previous analog study of university students even thought the samples were quite different, which may support the generalizability of previous analog studies. BEP and EMDR were the treatments chosen least often as a top choice and they were the forms of psychotherapy participants reported most wanting to avoid. Medication was listed as the least wanted intervention overall. Notably $60 \%$ of participants, compared to $57 \%$ for medication, selected EMDR as their least or second least choice. This is interesting because the results for EMDR are consistent with those found in Becker et al. (2007) and Tarrier et al. (2006). Thus, although EMDR appears popular among therapists, when study participants are given information that should be provided in a thorough informed consent process (i.e., clients are provided with the information they need about all viable treatment procedures and scientific support for these procedures so as to make an informed choice about treatment), EMDR is not selected over less controversial treatments with well established evidence bases. Similarly, although the literature suggests some clinician interest in using BEP to treat PTSD in police officers, this treatment also was not preferred to EBT in our sample. Interestingly, the major qualitative finding of this study was that law enforcement officers and cadets reported being very grateful that someone had finally asked them about their opinions about treatment. Thus, we encourage departments and therapists to seriously consider the preferences shown in this study, particularly given that there is an alignment between preferences and treatments with a strong evidence base. 
In addition to investigating opinions about PTSD treatment, we also asked participants to rate the credibility of CISD, to indicate how likely CISD would be to reduce onset of PTSD, and whether CISD should be mandated. The results may provide some insight into why CISD remains popular even though it may not only be ineffective, but also potentially harmful (Lilienfeld, 2007). More specifically, two studies to date have found negative effects when CISD was compared to assessment-only control at longer-term follow-up (Lilienfeld).

In this study, participant responses to the question about CISD's ability to reduce onset of PTSD indicated marked ambivalence about the efficacy of CISD given that the median rating on the 7-point Likert scale was 4.00 . Just under $20 \%$ of participants thought that CISD was very likely to reduce onset of PTSD and just over $25 \%$ thought it was very unlikely to reduce onset. Despite ambivalence about the efficacy of CISD, $77 \%$ of participants thought it should be required. We can think of two reasons for this discrepancy. First, CISD is widely used and has appealing features that are unrelated to efficacy (e.g., no stigma if all are required to attend post-trauma; gives appearance of meeting organizational duty of care requirements; conveys to officers that someone cares about them, uses peer-processes, provides screening opportunity). Second, participants rated the rationale for CISD as highly credible. Indeed, it was the only rationale rated as credible as exposure. Thus, the rationale for CISD may be so credible that it overrides evidence showing that it is not effective. Along this line, Lilienfeld (2007) notes that most people who receive CISD believe that it is helpful, even when objective measures show this is not the case. One option for reducing use of CISD may be to find strategies to maintain perceived benefits while eliminating the likely problematic component (i.e., forced debriefing). For instance, we conducted a very informal survey of a small number of police psychologists in major cities in Texas. Results indicated that a number had moved away from debriefing during required "CISD" meetings and instead simply provided supportive psychoeducation.

This study has a several limitations to. First, most participants did not have PTSD, although almost $50 \%$ had experienced a Criterion A event. Thus, further research is still needed 
with clinical samples. It should be noted, however, that results were largely consistent between the total sample and sub-groups who had experienced a Criterion A event or met criteria for PTSD. Second, it could be argued that responses were biased because some descriptions discussed scientific support to a greater degree than other descriptions. These differences, however, accurately represent what is known about the treatments. Thus, it would be inaccurate to claim that all interventions had the same level of scientific support. Third, because patients likely do not receive a complete listing of the empirical bases of multiple treatments in clinical settings, providing participants with this information in this study may challenge the ecological validity. We would argue, however, that perhaps patients should receive this information as part of a true informed consent process, and that this might create more grass roots interest in EBT. Fourth, although word lengths were roughly similar, they were not identical. Word length, however, did not appear to markedly influence choices. When examining the seven intervention descriptions, the three least chosen treatments had the longest (BEP), the shortest (sertraline), and the middle (EMDR) treatment lengths (i.e., three interventions had more words than EMDR and three had fewer words). The three most selected treatments had the second shortest (exposure), the second longest (CPT), and third longest descriptions (psychodynamic). Furthermore, CPT, psychodynamic therapy and EMDR were within a five word range. In summary, this study supports previous research showing that exposure and CPT for PTSD may be preferred over interventions with less scientific support or that are associated with greater controversy (e.g., EMDR). This study also provides evidence that law enforcement organizations could benefit from directing greater resources towards offering both CPT and exposure therapy for PTSD, which were collectively preferred by approximately $90 \%$ of participants as a first or second choice treatment. Provision of EBT for PTSD to law enforcement officers also may improve the palatability of treatment compared to other therapies that may be more available but less preferred and less efficacious. 


\subsection{References}

Addis, M. E., \& carpenter, K. M. (1999). Why, why, why?: Reason giving and rumination as predictors of response to activation- and insight oriented treatment rationales. Journal of Clinical Psychology, 55(7), 881-894.

Adler, A. B., Litz, B. T., Castro, C. A., Suvak. M., Thomas, J. L., Burrell, L., McGurk, D. Wright, K. M., \& Bliese, P. D. (2008). A group randomized trial of critical incident stress debriefing provided to U.S. peacekeepers. Journal of Traumatic Stress, 21, 253-263.

Angelo, F. N., Miller, H. E., Zoellner, L. A., \& Feeny, N. C. (2008). “I need to talk about it”: A qualitative analysis of trauma-exposed owmen's reasons for treatment choice. Behavior Therapy, 39 (1), 13-21.

Artwohl, A., \& Christensen, L. W. (1997). Deadly force encounters: What cops need to know to mentally and physically prepare for and survive a gunfight. Boulder, CO: Paladin Press.

Becker, C. B., Darius, E., Schaumberg, K. (2007). An analog study of patient preferences for exposure versus alternative treatments for posttraumatic stress disorder. Behaviour Research \& Therapy, 45, 2861-2873.

Becker, C. B., Zayfert, C., \& Anderson, E. (2004). A survey of psychologists' attitudes towards and utilization of exposure therapy for PTSD. Behaviour Research \& Therapy, 42, 277292.

Cahill, S. P., Foa, E. B., Hembree, E. A., Marshall, R. D., \& Nacash, N. (2006). Dissemination of exposure therapy in the treatment of posttraumatic stress disorder. Journal of Traumatic Stress, 19 (5), 597-610.

Devilly, G. J., Gist, R. \& Cotton, P. (2006). Ready! Fire! Aim! The status of psychological debriefing and therapeutic interventions: In the work place and after disasters. Review of General Psychology, 10 (4), 318-345. 
Dowling, F. G., Moynihan, G. \& Genet, B. (2006). A peer-based assistance program for officers with the New York City Police Department: Report of the effects of Sept. 11, 2001. American Journal of Psychiatry, 63, 151-153.

Foa, E. B., Cashman, L., Jaycox, L., \& Perry, K. (1997). The validation of a self-report measure of posttraumatic stress disorder: The Posttraumatic Diagnostic Scale. Psychological Assessment, 9(4), 445-451.

Foa, E. B., Keane, T. M., \& Friedman, M. J. (Eds.). (2000). Practice guidelines from the international society for traumatic stress studies: Effective treatments for PTSD. New York: The Guilford Press.

Foy, D. W., Kagan, B., McDermott, C., Leskin, G., Sipprelle, R. C., \& Paz, G. (1996). Practical parameters in the use of flooding for treating chronic PTSD. Clinical Psychology and Psychotherapy, 3(3), 169-175.

Freiheit, S. R., Vye, C., Swan, R. \& Cady, M. (2004). Cognitive-behavioral therapy for anxiety: Is dissemination working? Behavior Therapist, 27, 25-32.

Gersons, B. P. R., Carlier, I. V. E., Lamberts, R. D., \& van der Kolk, B. A. (2000). Randomized clinical trial of brief eclectic psychotherapy for police officers with posttraumatic stress disorder. Journal of Traumatic Stress, 13(2), 333-347.

Gist, R., \& Devilly, G. J. (2002) Post-trauma debriefing: the road too frequently traveled. The Lancet, 360,741-742.

Kates, A. R. (1999). CopShock: Surviving post-traumatic stress disorder (PTSD). Tucson, AZ. Holbrook Street Press.

Klinger, D. (2006). Into the Kill Zone: A cop's eye view of deadly force. San Francisco, CA: Jossey-Bass.

Lilienfeld, S. O. (2007). Psychological treatments that cause harm. Perspectives on Psychological Science, 2(1), 53-70. 
Lindauer, R. L., Gersons, B. P., van Meijel, E. P., Blom, K., Carlier, I. Vrijlandt, V. I. \& Olff, M. (2005). Effects of brief eclectic psychotherapy in patients with Posttraumatic Stress Disorder: Randomized clinical trial. Journal of Traumatic Stress, 18, 205-212.

Miller, L. (2006). Practical police psychology. Springfield, IL: Charles C. Thomas, Publisher.

Najavits, L. M. (2006). Present- versus past-focused therapy for posttraumatic stress disorder/substance abuse: A study of clinician preferences. Brief Treatment and Crisis Intervention, 6(3), 248-254.

Resick, P. A., Nishith, P., Weaver, T. L., Astin, M. C., \& Feuer, C. A. (2002). A comparison of cognitive-processing therapy with prolonged exposure and a waiting condition for the treatment of chronic posttraumatic stress disorder in female rape victims. Journal of Consulting and Clinical Psychology, 70(4), 867-879.

Resick, P. A., \& Schnicke, M. K. (1992). Cognitive processing therapy for sexual assault victims. Journal of Consulting and Clinical Psychology, 60, 748-756.

Rosen, C. S., Chow, H.S., Finney, J.F., Greenbaum, M.A., Moos, R.H., Javaid, I.S., Yesavage, J.A. (2004). VA practice patterns and practice guidelines for treating posttraumatic stress disorder. Journal of Traumatic Stress, 17, 213-222.

Rothbaum, B. O., Astin, M. C., Marsteller, F. (2005). Prolonged exposure versus eye movement desensitization and reprocessing (EMDR) for PTSD rape victims. Journal of Traumatic Stress, 18, 607-616.

Tarrier, N., Liversidge, T., Gregg, L. (2006). The acceptability and preference for the psychological treatment of PTSD. Behaviour Research and Therapy, 44(11), 1643-1656.

van Emmerik, A. A., Kamphuis, J. H., Hulsbosch, A. M., \& Emmelkamp, P. M. (2002). Single session debriefing after psychological trauma: a meta-analysis. The Lancet, 360, 766771. 
Volanti, J. M., Andrew, M. E., Burchfiel, C. M., Dorn, J., Hartley, T., \& Miller, D. B. (2006). Posttraumatic stress symptoms and subclinical cardiovascular disease in police officers. International Journal of Stress Management, 13 (4), 541-554.

Wright, L., Borrill, J., Teers, R., \& Cassidy, T. (2006). The mental health consequences of dealing with self-inflicted death in custody. Counseling Psychology Quarterly, 19 (2), 165-180.

Young, J., Connolly, K., \& Lohr, J. M. (2008). Fighting the good fight by hunting the dodo bird to extinction: ABCT's dissemination effort. Behavior Therapist, 31(5), 97-100.

Zoellner, L. A., Feeny, N. C., Cochran, B., \& Pruitt, L. (2003). Treatment choice for PTSD. Behaviour Research \& Therapy, 41, 879-886. 
Acknowledgments: We are grateful to Captain Charlie Conn, Steubenville Police Department; Rudy A. Gonzalez and David Deluna, Trinity University; and Chief William McManus, San Antonio Police Department for their support of this study.

Parts of this manuscript were presented at the $116^{\text {th }}$ Annual Convention of the American Psychological Association, Boston, MA. 
Table 1. Percent of participants who chose each therapy option as most or second most

\begin{tabular}{|c|c|c|c|c|c|c|}
\hline & \multicolumn{2}{|c|}{$\begin{array}{c}\text { Total Sample } \\
(N=379)\end{array}$} & \multicolumn{2}{|c|}{$\begin{array}{l}\text { Criterion A } \\
(n=218)\end{array}$} & \multicolumn{2}{|c|}{$\begin{array}{c}\text { PTSD } \\
(n=36)\end{array}$} \\
\hline & $\begin{array}{c}\text { Top Choice } \\
(\%)\end{array}$ & $\begin{array}{c}\text { Top } 2 \\
(\%)\end{array}$ & $\begin{array}{c}\text { Top Choice } \\
(\%)\end{array}$ & $\begin{array}{c}\text { Top } 2 \\
(\%)\end{array}$ & $\begin{array}{c}\text { Top Choice } \\
(\%)\end{array}$ & $\begin{array}{c}\text { Top } 2 \\
(\%)\end{array}$ \\
\hline CPT & 36.9 & 56.7 & 39.0 & 58.7 & 38.9 & 61.1 \\
\hline Exposure & 25.9 & 58.8 & 20.6 & 59.2 & 11.1 & 52.7 \\
\hline Psychodynamic & 13.2 & 29.0 & 14.2 & 28.9 & 25.0 & 36.1 \\
\hline Sertraline & 9.2 & 21.6 & 10.1 & 19.7 & 8.3 & 19.4 \\
\hline BEP & 9.0 & 20.8 & 10.1 & 22.0 & 13.9 & 27.7 \\
\hline EMDR & 1.8 & 5.8 & 2.3 & 5.0 & 2.8 & 2.8 \\
\hline
\end{tabular}

Note: Criterion $\mathrm{A}=$ all participants reporting lifetime occurrence of a criterion $\mathrm{A}$ event. PTSD = all participants meeting full criteria for PTSD. CPT = Cognitive Processing Therapy. BEP $=$ Brief Eclectic Psychotherapy. EMDR = Eye Movement Desensitization Reprocessing. 
Table 2. Mean, standard deviation, range on credibility scale and PDS

\begin{tabular}{|c|c|c|c|}
\hline Measure & $M$ & $S D$ & Range \\
\hline CS - Exposure & 32.89 & 8.69 & $7-49$ \\
\hline CS - CISD & 31.29 & 9.68 & $7-49$ \\
\hline $\mathrm{CS}-\mathrm{CPT}$ & 31.26 & 8.17 & $7-49$ \\
\hline CS - BEP & 27.15 & 9.62 & $7-49$ \\
\hline CS - Psychodynamic & 26.79 & 10.26 & $7-49$ \\
\hline CS - Sertraline & 22.77 & 9.82 & $7-49$ \\
\hline CS - EMDR & 18.53 & 9.32 & $7-49$ \\
\hline PDS symptom severity ${ }^{a}$ & 7.61 & 8.90 & $0-42$ \\
\hline PDS diagnosis & $9.50 \%$ & $\mathrm{~N} / \mathrm{A}$ & $\mathrm{N} / \mathrm{A}$ \\
\hline \multicolumn{4}{|c|}{ Note: $\mathrm{N}=379 .{ }^{a}$ Symptom severity is calculated for those reporting a Criterion A event $(n=69)$. } \\
\hline
\end{tabular}




\section{Appendix A}

Cognitive Processing Therapy (219 words): Cognitive processing therapy (CTP) is a treatment for PTSD. CPT can be done in individual or groups and typically takes 12 sessions. CPT has undergone rigorous scientific evaluation and has been supported by clinical trials. The goal of CPT is for you to "process" emotions and confront your beliefs about the incident and its effects on your life. You want to make sense of your traumatic experience. CPT is a form of cognitive behavioral therapy and is used for many traumatic incident related problems.

Procedures used include:

- You will receive education about traumatic events.

- You also will be asked to write detailed stories of the traumatic event and will read them aloud in session and for homework.

- You will be provided basic education about feelings and given information about how self-statements or thoughts affect how you feel

- You will learn how to identify 'stuck points' (troubling emotions from the traumatic event)

- You will learn to challenge incorrect beliefs about the traumatic event such as self blame.

- You will find and maintain a balanced, realistic perception of the world.

If you choose CPT, you will complete weekly 90-minute individual therapy sessions. You will not receive medication for your PTSD symptoms. Side effects of CPT include uncomfortable feeling and unsettling thoughts when remembering the trauma.

Medication treatment-Zoloft (192 words): Antidepressants medications are one of the available treatments for PTSD. Zoloft (Setraline) has undergone some of the most rigorous scientific evaluation; it is the only FDA approved medication for the treatment of PTSD. Zoloft is a type of 
antidepressant called a selective serotonin reuptake inhibitor. It has fewer side effects than older medicines used to treat PTSD.

In this treatment:

- You do not talk a great deal about your traumatic experience or be asked to confront situations or places you are avoiding.

- You see a psychiatrist weekly for general encouragement and to check out how the medication is working and look for side effects

- Your medication will be adjusted if need be after examination.

- At the end of 10 weeks, the medication will be reduced gradually to minimize the chance of withdrawal symptoms.

If you choose this medicine, you will take up to $200 \mathrm{mg}$ of Zoloft on a daily basis for 10 weeks. The risks from Zoloft are mild to moderate side effects or withdrawal symptoms. Possible side-effects include loose stools, sweating, nausea, headache, fatigue, anorexia, weight loss or gain, sexual impairment, increased anxiety, restlessness, and insomnia.

Brief Eclectic Psychotherapy (228 words): Brief Eclectic Psychotherapy (BEP) is a newer therapy used for traumatic symptoms. It uses different scientifically-supported treatments that have each been shown to be effective in treating PTSD. BEP combines parts of cognitivebehavioral and psychodynamic therapy to form a brief, more effective way to treat these symptoms. Since BEP is new, it has not been rigorously tested. However, because it uses methods which used in reducing PTSD symptoms, it could be an effective treatment.

This treatment consists of:

- Individual psychotherapy starting with psychological education about the symptoms of PTSD and treatment protocols 
- Imagining the memory of the event concentrating on sensory details of the experience

- Writing assignments such a daily diary or writing letters to persons or institutions involved in the trauma

- Learning to understand the fundamental changes in one's life after trauma and developing meaning

- A farewell ritual to fully express any remaining sorrow over the event, realize that you have left the event behind you and have gained control of your life.

Each part of this treatment is designed to allow you to regain control and predictability of your everyday life, and decrease your PTSD symptoms. If you choose this treatment, you will meet with a therapist for one hour a week for 16 weeks. You will not receive medication. Risk for BEP could include limited effectiveness for acute PTSD symptoms.

Eye Movement Desensitization and Reprocessing (214 words): Eye Movement Desensitization and Reprocessing (EMDR) is a structured form of psychotherapy that uses ideas from other treatments. Some research supports the use of EMDR. EMDR generally has positive outcomes and may sometimes be used in combination with other therapies. It has you focus on external stimuli, such as a moving visual object, and tells you to remember traumatic incidents. External stimuli may be tapping with each hand or having your therapist move his/her finger back and forth in front of your eyes.

The therapy goes as follows:

- You will think of the most vivid memory of your trauma and a negative idea.

- You will focus on the memory image while moving your eyes back and forth and following the therapist's finger.

- You will be asked to monitor your body's sensations 
- You are then instructed to let your mind wander and/or go blank. Next, you will catalogue your mind's activities.

- Next, your therapist will help you identify the next therapy target. You will simultaneously work on increasing positive ideas.

If you choose EMDR, you will meet with a therapist for several sessions. Often, the number of sessions will be quite limited. You will not receive medications. Risks can include mental distress which can cause adverse arousal of the body.

Exposure Therapy (205 words): Exposure Therapy (ET) is a 9-12 session individual therapy for PTSD. ET has been shown to be effective in the treatment of PTSD, and has been thoroughly and scientifically tested. Several controlled studies have shown it to significantly reduce PTSD symptoms. ET is a type of cognitive behavioral treatment that targets a number of traumarelated difficulties.

Procedures for this treatment include:

- Education about common reactions to trauma,

- Breathing retraining (relaxation training),

- Prolonged (repeated) exposure to trauma memories,

- Repeated in vivo (i.e. real life) exposure to situations that you are avoiding due to trauma related fear.

In other words, you will be encouraged to confront the traumatic memory by repeatedly telling the story to the therapist and to challenge things in your life that you are avoiding because you are afraid (i.e. driving a car, walking home at night). You will be assigned homework to encourage you to practice in life the things you learn in therapy. 
If you choose to receive ET treatment, you will meet with a therapist about once a week for 60-90 minutes. You will not be prescribed any medication. The risks associated with ET are mild to moderate discomfort when exposed to anxiety-provoking images, situations, and places.

Psychodynamic Therapy (216 words): Psychodynamic therapy has been used for over a 100 years to treat traumatic symptoms. The goal is to what understand what happened in the traumatic event and any hidden memories. You want to make sense of defensive psychological processes that allow your unconscious and repressed memories to cause pathological symptoms. Psychodynamic therapy generally has not been rigorously evaluated in research trials because treatment tends to focus on psychological processes as opposed to people's symptoms.

The therapeutic relationship is a critical component of therapy. The goals and procedures are:

- To help you learn how to manage your intense emotions.

- You also will work to achieve a balance between your subjective needs, the external demands of the world, and your traumatic memories.

- Your therapist generally will maintain a neutral attitude during therapy and will not give you advice regarding what to do.

- Instead, will explore you feelings and behaviors so that you can gain insight about your symptoms.

If you chose psychodynamic psychotherapy, you will meet individually with a therapist who will help you work through your underlying issues so that you are able to understand the meaning of your unconscious processes. Treatment may be long term. You will not receive medication for your PTSD symptoms. Side effects may include uncomfortable and unsettling thoughts. 
Critical Incident Stress Debriefing (207 words): Critical Incident Stress Debriefing (CISD) is a treatment requiring only one or two intervention sessions of debriefing right after a traumatic event. A debriefing process lets the person involved with the traumatic event process and understand the incident's impact. CISD is defusing which allows the discussion of thoughts and emotions from the traumatic incident (called defusing). CISD typically occurs as soon as possible following the incident but no longer than 24-72 hours after the event. Supporters of CISD say that people who receive CISD within this 24-72 hour time period have reduced shortterm and long-term crisis reactions or psychological trauma. Recent research, however, has shown that CISD may not have long-term effectiveness.

In the CISD intervention session:

- You will discuss the impact of the traumatic event

- You will also discuss the thoughts, emotions, and feelings surrounding the occurrence as well as possible future reactions

- Finally, you will be asked to attempt to bring closure to the event and discuss ways to move forward

If you choose this intervention method, you will receive a one time debriefing/defusing session immediately following the shooting. You will not receive medication. There are few documented side effects of CISD. However, there are some reports of increased stress after CISD. 